\title{
Stretched shadings and a Banach measure that is not scale-invariant
}

\author{
by \\ Richard D. Mabry (Shreveport, LA)
}

\begin{abstract}
It is shown that if $A \subset \mathbb{R}$ has the same constant shade with respect to all Banach measures, then the same is true of any similarity transformation of $A$ and the shade is not changed by the transformation. On the other hand, if $A \subset \mathbb{R}$ has constant $\mu$-shade with respect to some fixed Banach measure $\mu$, then the same need not be true of a similarity transformation of $A$ with respect to $\mu$. But even if it is, the $\mu$-shade might be changed by the transformation. To prove such a $\mu$ exists, a Hamel basis with some weak closure properties with respect to multiplication is used to construct sets with some convenient scaling properties. The notion of shade-almost invariance is introduced, aiding in the construction of a variety of Banach measures, in particular, one that is not scale-invariant.
\end{abstract}

1. Introduction. Let $\mathfrak{L}$ denote the family of Lebesgue measurable subsets of the set $\mathbb{R}$ of real numbers and let $\lambda$ denote the usual Lebesgue measure on $\mathfrak{L}$.

A Banach measure on $\mathbb{R}$ is an isometry-invariant total extension of $\lambda$, i.e., an isometry-invariant extension of $\lambda$ defined on $2^{\mathbb{R}}$. It is well known that Banach measures exist as a consequence of the axiom of choice and that they are only finitely additive. (In this paper, unless explicitly noted, a measure will be assumed to be only finitely additive.) Let $\mathcal{B}$ denote the class of all Banach measures on $\mathbb{R}$.

It is also known that Banach measures on $\mathbb{R}$ exist that are scale-invariant (see [19, Cor. 11.5] and [9]). What seems not to be known up to now, or at least not mentioned, is whether Banach measures exist which are not scaleinvariant. That such measures do indeed exist is the main result of this paper (Section 10).

Of course the Lebesgue measure $\lambda$ is scale-invariant; the question is whether or not Banach measures should inherit that property directly from $\lambda$

2010 Mathematics Subject Classification: Primary 28A12; Secondary 28A05.

Key words and phrases: almost independence, almost invariance, Archimedean set, Banach measure, Hamel basis, invariant ideal, Lebesgue measure, multiplicative extension, scaleinvariance, shade-almost invariance, shading, tower. 
or perhaps have it as a consequence of isometry-invariance. In [9] it is shown that scale-invariant Banach measures do exist (in $\mathbb{R}^{2}$ as well as $\mathbb{R}$; see also [13] and [19, Cor. 11.5]) but there is no corresponding proof or suggestion that Banach measures do not come already equipped with this property. By comparison, the Lebesgue measure on $\mathbb{R}^{n}$ gets its scale-invariance gratis - it is the unique translation-invariant countably additive measure defined on the Lebesgue measurable sets and normalizing $[0,1]^{n}$; its invariance with respect to all other isometries and its respect for similarity are natural consequences. But this is not the case for Banach measures, as they lack countable additivity. This sort of thing is noted following Theorem 11.21 in [19], where it is pointed out that there exist total extensions of $\lambda$ which are translation-invariant but not reflection-invariant. (A more general result is proved in [18.)

To see how much we can stretch things, we try to be somewhat general where convenient, obtaining examples of Banach measures in which scaleinvariance is violated in different, fairly customizable fashions. To do this we exploit properties of sets we call shade-almost invariant. Our examples of such sets are created using Hamel bases, their shade-almost invariance being used to extend $\lambda$, creating large families of distinct Banach measures. By giving our Hamel bases some additional algebraic properties, we make our resulting Banach measures have some convenient properties with respect to scaling. Along the way, we explore some interesting behavior of shadings under similarity transformations.

Most of our set-theoretic notation is fairly standard. The rationals and integers are denoted by $\mathbb{Q}$ and $\mathbb{Z}$, respectively. The cardinality of a set $X$ is denoted by $|X|$. The cardinality of the continuum is denoted by $c$, as is the least ordinal with this cardinality. If $X$ and $Y$ are subsets of reals, we let $X+Y$ denote $\{x+y: x \in X, y \in Y\}$. When $x \in \mathbb{R}, x+Y$ is synonymous with $\{x\}+Y$. Similarly, $X-Y$ and $x-Y$ have the obvious meanings, $x Y=\{x y: y \in Y\}$, and combinations of these notations are freely used. Symmetric differences of sets are indicated by $\triangle$ and we emphasize disjoint unions with $\uplus$.

The term "scale-invariance" has the traditional meaning here. We shall deal exclusively with subsets of the real line, so for us a measure $m$ is scaleinvariant if $m(h S)=|h| m(S)$ whenever $h \in \mathbb{R}$ and $S$ is $m$-measurable.

\section{Basic properties of shadings}

Definition 2.1. For a fixed $t \in[0,1]$, a subset $A$ of $\mathbb{R}$ is said to be a shading of $\mathbb{R}$, and to have shade (or shade-density) equal to $t$, if

$$
\mu(A \cap E)=t \mu(E)
$$


for every bounded $E \in \mathfrak{L}$ and every $\mu \in \mathcal{B}$. We then write $\operatorname{sh}(A)=t$. (This notion was introduced in [10].)

We will show (Theorem 4.1) that if $A \subset \mathbb{R}$ has constant shade and $g: \mathbb{R} \rightarrow \mathbb{R}$ is any similarity transformation, then $\operatorname{sh}(g(A))=\operatorname{sh}(A)$. Thus, a stretch does not affect constant shade-density. This is easy to prove and has an intuitive appeal (if intuition can be brought to bear on such shady consequences of the axiom of choice). Supposing, for instance, that $A$ has shade $1 / 3$, we may think of $A$ as taking up $1 / 3$ of each interval, no matter how small. Stretching $A$ uniformly by any amount should not change that. But we shall see that this view (which is only heuristic) depends also on the "uniformity with respect to $\mu$ " in the definition of shade. We will make the last statement more precise (it is the main point of this article) in Section 4

Definition 2.2. For a fixed $\mu \in \mathcal{B}$ we call $A \subset \mathbb{R}$ a $\mu$-shading with $\mu$-shade equal to $t$, and write $\operatorname{sh}_{\mu}(A)=t$, if $\mu(A \cap E)=t \mu(E)$ for each bounded $E \in \mathfrak{L}$. (To be more specific, $A$ has constant $\mu$-shade in this case.)

It is clear that $\operatorname{sh}(A)=t$ iff $\operatorname{sh}_{\mu}(A)=t$ for all $\mu \in \mathcal{B}$.

In the definitions of shading and $\mu$-shading, it is equivalent to require that (1) hold whenever $E$ is a bounded interval. (This is explicitly shown for shadings in 10 - see, in particular, Definition 3.7 in conjunction with Theorem 3.11 there - but easily extends to $\mu$-shadings.) In this paper we will mainly concern ourselves with sets of constant shade or $\mu$-shade, but it is possible for a set to have, for example, smoothly varying shade. In fact, for any continuous $\varphi: \mathbb{R} \rightarrow[0,1]$, there exists a set $F \subset \mathbb{R}$ having the property that

$$
\lim _{\mu(J) \rightarrow 0} \mu(F \cap J) / \mu(J)=\varphi(x)
$$

for every $\mu \in \mathcal{B}$, where $J$ represents a bounded interval containing $x$. It then makes sense to write $\operatorname{sh}(F)=\varphi$ (see Thm. 5.7 in [10]). Similarly, for a fixed $\mu \in \mathcal{B}$, the notation $\operatorname{sh}_{\mu}(F)=\varphi$ has the obvious meaning. Shade is an extension of the usual Lebesgue density.

For other basic properties of shadings, see [10]. For an introduction to the main ideas and motivations behind various extensions of Lebesgue measure, see [2] or [3]. For an extensive look, see [19] or [7].

3. Shadings versus $\mu$-shadings. There are examples of sets that show the distinction between shadings and $\mu$-shadings is not vacuous.

Definition 3.1. A set $A \subset \mathbb{R}$ is called Archimedean if there is a dense set of translators $\{x\}$ for which $A+x=A$. (This term was coined in [17].)

It is easy to prove (as in [10, Thm. 6.1]; see also Corollary 5.4 in the present paper) that for every Archimedean set $A$ and every $\mu \in \mathcal{B}$, the set $A$ 
has constant $\mu$-shade. (See [14 for more results concerning Banach measures and Archimedean sets.)

Definition 3.2. Let $G$ be a group of transformations on $\mathbb{R}$. A set $A \subset \mathbb{R}$ is called almost $G$-invariant if $|A \triangle f(A)|<c$ for every $f \in G$. The set $A$ is simply called almost invariant when $G$ is the group of all isometries on $\mathbb{R}$. (See, e.g., [7, p. 9] or [8, p. 76].)

It is easy to prove (Corollary 5.5) that if $A \subset \mathbb{R}$ is almost invariant, then $A$ has constant $\mu$-shade for any fixed $\mu \in \mathcal{B}$. (It suffices for $A$ to be almost $G$-invariant when $G$ contains a dense set of translations.)

So Archimedean sets and almost invariant sets are "uniformly dense" with respect to any fixed $\mu \in \mathcal{B}$. But it turns out that in either of these two classes of sets, there exist a set $A$ and Banach measures $\mu_{1}, \mu_{2}$ for which $\operatorname{sh}_{\mu_{1}}(A) \neq \operatorname{sh}_{\mu_{2}}(A)$, so neither Archimedean sets nor almost invariant sets are guaranteed to be shadings (although they can be). Hence one cannot reasonably associate any specific, fixed density (in our sense, at least) with such $A$. Indeed, this sort of situation can be extreme - in [6 an almost invariant set $C \subset \mathbb{R}$ is constructed, along with a family $\left\{\nu_{t}\right\}_{t \in[0,1]}$ of isometry-invariant countably additive extensions of $\lambda$, for which $\nu_{t}(C \cap E)=t \lambda(E)$ for each $t$ and for any bounded $E \in \mathfrak{L}$. Since each such $\nu_{t}$ can be extended to a Banach measure $\mu_{t}$, we have $\operatorname{sh}_{\mu_{t}}(C)=t$ for each $t \in[0,1]$. (For the set $C$, take any of the sets denoted by $A_{\gamma}$ in [6]; see the final paragraphs in that paper.) Although not originally constructed to be so, this $C$ can easily be made Archimedean. Indeed, for any set $B \subset \mathbb{R}$, the set $B+\mathbb{Q}$ is Archimedean with dense set of translators $\mathbb{Q}$. If $B$ is almost invariant then so is $B+\mathbb{Q}$, and furthermore, $|(B+\mathbb{Q}) \triangle B|<c$. The shade of any noncontinuum set is zero ([10, Lemma 4.5]) so $\operatorname{sh}_{\mu}(B+\mathbb{Q})=\operatorname{sh}_{\mu}(B)$ for any $\mu \in \mathcal{B}$. Therefore, if $C$ is the example in [6], the set $C+\mathbb{Q}$ is both Archimedean and almost invariant and has the property that for each $t \in[0,1]$ there exists a $\mu_{t} \in \mathcal{B}$ for which $\operatorname{sh}_{\mu_{t}}(C+\mathbb{Q})=t$.

At the other extreme, Neu proves in [15, Thm. 2.2] that if $S$ is an almost isometry-invariant shading, then $\operatorname{sh}(S)=0$ or 1 .

4. The main problem. Keith Neu asked (private communication) the following, circa 2004: Is the $\mu$-shade of a $\mu$-shading necessarily preserved under a similarity transformation? That is, is it true that $\operatorname{sh}_{\mu}(g(A))=\operatorname{sh}_{\mu}(A)$ whenever $A$ is a $\mu$-shading and $g$ is a similarity transformation? We answer this question in the negative (Theorem 10.1). Our counterexample will be Archimedean but not almost invariant. A few years after reading an earlier draft of this paper, Neu (private communication) devised a slick counterexample that is almost invariant. 
Specifically, we will show the existence of a Banach measure $\mu$, an Archimedean set $A \subset \mathbb{R}$ and $h>0$ such that $\operatorname{sh}_{\mu}(A) \neq \operatorname{sh}_{\mu}(h A)$. We shall see that the pair of numbers $\operatorname{sh}_{\mu}(A)$ and $\operatorname{sh}_{\mu}(h A)$ can be any values at all in $[0,1]$ for fairly arbitrary values of $h$.

First, in the opposite direction, we prove an easy but revealing result, namely that if a set $A$ has constant shade $t$, then so does any similarity transformation of $A$. We prove a slightly stronger result because it is not much extra trouble.

THEOREM 4.1. If $g$ is a similarity transformation on $\mathbb{R}$ and $F$ is a $\varphi$ shading of $\mathbb{R}$, where $\varphi: \mathbb{R} \rightarrow[0,1]$, then $\operatorname{sh}(g(F))=\varphi \circ g^{-1}$. In particular, if $\varphi(x)=t$ is a constant function, then $\operatorname{sh}(g(F))=\operatorname{sh}(F)=t$.

Proof. Let $\mu \in \mathcal{B}$ and let $g(x)=a x+b$ for reals $b$ and $a \neq 0$. For $X \subset \mathbb{R}$, consider the set function $\mu_{g}$ defined by

$$
\mu_{g}(X)=\frac{1}{|a|} \mu(g(X))=\frac{1}{|a|} \mu(a X+b)=\frac{1}{|a|} \mu(a X) .
$$

It is easy to see that $\mu_{g}$ extends Lebesgue measure, since $\mu$ itself does: if $E \in \mathfrak{L}$, then $g(E) \in \mathfrak{L}$ and $\lambda(g(E))=|a| \lambda(E)$, hence $\mu_{g}(E)=(1 /|a|) \mu(a E)$ $=(1 /|a|) \lambda(a E)=\lambda(E)$. Also, the isometry-invariance of $\mu$ easily yields the isometry-invariance of $\mu_{g}$, so $\mu_{g}(f(X))=\mu_{g}(X)$. The fact that $\mu$ is defined for all subsets of $\mathbb{R}$ yields the same for $\mu_{g}$, hence $\mu_{g}$ is itself a Banach measure. As such, it must be that (2) holds with $\mu_{g}$ in place of $\mu$, since $F$ is a $\varphi$-shading. Fixing a bounded interval $J$ and $x \in J$, we have

$$
\begin{aligned}
\frac{\mu(g(F) \cap J)}{\mu(J)} & =\frac{\mu\left(g\left(F \cap g^{-1}(J)\right)\right)}{\mu(J)}=\frac{|a| \mu_{g}\left(F \cap g^{-1}(J)\right)}{\mu(J)} \\
& =\frac{|a| \mu_{g}\left(F \cap g^{-1}(J)\right)}{|a| \mu_{g}\left(g^{-1}(J)\right)} .
\end{aligned}
$$

Clearly, $g^{-1}(x) \in g^{-1}(J)$ and $\mu\left(g^{-1}(J)\right) \rightarrow 0$ as $\mu(J) \rightarrow 0$. Since $F$ is a $\varphi$ shading, the last term approaches $\varphi\left(g^{-1}(x)\right)$ as $\mu(J) \rightarrow 0$, and that finishes the proof.

The rest of this paper is devoted to showing that in the theorem just proved, shade cannot be replaced with $\mu$-shade. We will need some preliminaries first.

5. Shade-almost invariance. Denote by $\mathcal{Z}$ the class of sets having shade zero in $\mathbb{R}$. It is clear that $\mathcal{Z}$ is an ideal in $\mathbb{R}$, and that $\mathcal{Z}$ is $G$-invariant when $G$ is the group of isometries on $\mathbb{R}$. We also note that $\mathcal{Z}$ is not a $\sigma$-ideal. One can see this via a set $W\left(h_{0}\right)$, defined later (Definition 7.1), having the following property: there is a positive number $h_{0}$ such that for any $q \in \mathbb{Q}$, $q h_{0}+W\left(h_{0}\right) \in \mathcal{Z}$ but $\bigcup_{q \in \mathbb{Q}}\left(q h_{0}+W\left(h_{0}\right)\right)=\mathbb{R} \notin \mathcal{Z}$. 
Definition 5.1. For subsets $A$ and $B$ of $\mathbb{R}$, we write $A \doteqdot B$ if $A \triangle B \in \mathcal{Z}$. We will then say that $A$ and $B$ are shade-almost equal. (One could also say that $A$ and $B$ are "not a shade different".) Two sets $A$ and $B$ will be called shade-almost disjoint if $A \cap B \doteqdot \emptyset$.

Clearly, $A \doteqdot \emptyset$ iff $A \in \mathcal{Z}$. We do not make use of it, but we note that the relation $\doteqdot$ is an equivalence relation on $\mathbb{R}$

Definition 5.2. For a group $G$ of isometries on $\mathbb{R}$, we say that $A$ is shade-almost $G$-invariant if $A \doteqdot g(A)$ for each isometry $g \in G$. If $G$ is the group of all isometries on $\mathbb{R}$, we simply say that $A$ is shade-almost invariant.

We observe that shade-almost invariance is weaker than almost invariance: as already noted, in [10, Lemma 4.5] it is shown that if $|Y|<c$ then $\operatorname{sh}(Y)=0$, but that the set $W\left(h_{0}\right)$ (Def. 7.1 again) has shade zero, while $\left|W\left(h_{0}\right)\right|=c$. We also note that for any shade-almost invariant set $A$ and Banach measure $\mu$, the $\mu$-shade of $A$ is constant. For completeness, we include a short, easy proof of this, using slightly weaker assumptions.

THEOREM 5.3. Suppose $G$ is a group of isometries on $\mathbb{R}$ that includes a dense set of translations. Let $\mu \in \mathcal{B}$ and suppose that $A$ is a shade-almost $G$-invariant subset of $\mathbb{R}$. Then $A$ has constant $\mu$-shade.

Proof. Recall that $\uplus$ denotes a disjoint union. Suppose $J_{1}$ and $J_{2}$ are any two nonempty bounded intervals in $\mathbb{R}$. We first assume that $\lambda\left(J_{1}\right)=\lambda\left(J_{2}\right)$. Let $0<\varepsilon<\lambda\left(J_{1}\right)$. Choose a translation $g \in G$ such that $\lambda\left(g\left(J_{1}\right) \triangle J_{2}\right)<\varepsilon$. Then

$$
\begin{aligned}
\mu\left(A \cap J_{1}\right) & =\mu\left(g\left(A \cap J_{1}\right)\right)=\mu\left(g(A) \cap g\left(J_{1}\right)\right) \\
& =\mu\left([(g(A) \cap A) \uplus(g(A) \backslash A)] \cap g\left(J_{1}\right)\right) \\
& =\mu\left((g(A) \cap A) \cap g\left(J_{1}\right)\right)+\mu\left((g(A) \backslash A) \cap g\left(J_{1}\right)\right) \\
& \leq \mu\left(A \cap g\left(J_{1}\right)\right)+\mu\left((g(A) \triangle A) \cap g\left(J_{1}\right)\right)=\mu\left(A \cap g\left(J_{1}\right)\right) \\
& \leq \mu\left(A \cap\left(J_{2} \cup g\left(J_{1}\right)\right)\right)=\mu\left(A \cap\left[J_{2} \uplus\left(g\left(J_{1}\right) \backslash J_{2}\right)\right]\right) \\
& =\mu\left(A \cap J_{2}\right)+\mu\left(A \cap\left(g\left(J_{1}\right) \backslash J_{2}\right)\right) \\
& \leq \mu\left(A \cap J_{2}\right)+\mu\left(g\left(J_{1}\right) \backslash J_{2}\right) \leq \mu\left(A \cap J_{2}\right)+\varepsilon,
\end{aligned}
$$

and it follows that $\mu\left(A \cap J_{1}\right) \leq \mu\left(A \cap J_{2}\right)$. A symmetric argument (obtained by reversing the roles of $J_{1}$ and $J_{2}$ and replacing $g$ with $g^{-1}$ ) shows that $\mu\left(A \cap J_{2}\right) \leq \mu\left(A \cap J_{1}\right)$. Therefore, we have $\mu\left(A \cap J_{1}\right)=\mu\left(A \cap J_{2}\right)$ when $\lambda\left(J_{1}\right)=\lambda\left(J_{2}\right)$.

In the case that the intervals $J_{1}$ and $J_{2}$ are of different sizes, let $\delta>0$ and cover each $J_{i}$ by letting $\biguplus_{k=1}^{N_{i}} I_{i}^{(k)} \subset J_{i} \subset \biguplus_{k=0}^{N_{i}} I_{i}^{(k)}$, where for each fixed $i=1,2$, the $I_{i}(k)$ are all half-open intervals of size $\delta$. From the previous case concerning intervals of equal length, we may choose $t \in[0,1]$ such that $t \delta=\mu\left(A \cap I_{i}^{(k)}\right)$ for each $i=1,2$ and $0 \leq k \leq N_{i}$. We obtain the following 
two inequalities:

$$
N_{i} t \delta \leq \mu\left(A \cap J_{i}\right) \leq\left(N_{i}+1\right) t \delta, \quad N_{i} \delta \leq \mu\left(J_{i}\right) \leq\left(N_{i}+1\right) \delta
$$

Dividing these gives

$$
\frac{N_{i}}{N_{i}+1} t \leq \frac{\mu\left(A \cap J_{i}\right)}{\mu\left(J_{i}\right)} \leq \frac{N_{i}+1}{N_{i}} t,
$$

and now letting $\delta \rightarrow 0$ (so that $N_{i} \rightarrow \infty$ for each $i=1,2$ ) leads to

$$
\frac{\mu\left(A \cap J_{i}\right)}{\mu\left(J_{i}\right)}=t,
$$

and that proves the claim.

Corollary 5.4. If $A$ is Archimedean and $\mu \in \mathcal{B}$, then $A$ is a $\mu$-shading.

Corollary 5.5. If $A$ is almost invariant and $\mu \in \mathcal{B}$, then $A$ is a $\mu$ shading.

The proofs of these two corollaries follow easily by choosing the appropriate $G$ in Theorem 5.3. In the case of Archimedean sets it is the dense set of translations corresponding to the definition of an Archimedean set. (As already mentioned, Corollary 5.4 is given in [10, Thm 6.1].) For almost invariant sets, $G$ is arbitrary, so long as it contains a dense set of translations; "almost invariant" can then be replaced by "almost $G$-invariant" in Corollary 5.5 .

In Section 6 we use the notion of shade-almost invariance to generate Banach measures with some convenient properties.

6. Shady extensions. One way to construct countably additive-isometry invariant extensions of $\lambda$ is to add to the domain $\mathfrak{L}$ of $\lambda$ those sets generated by $\mathfrak{L}$ and a fixed almost invariant set having inner measure zero and full outer measure. Such measures can then be extended to Banach measures. As we will see, something similar can be done using shade-almost invariant sets. But Banach measures already do so much that we need not always construct new ones from scratch when we want them to have certain properties - we can often start with "existing" ones.

Lemma 6.1. Suppose $B$ is a shade-almost invariant subset of $\mathbb{R}$ that does not have shade zero. Then there exists a $\nu \in \mathcal{B}$ for which $\operatorname{sh}_{\nu}(B)=1$.

Proof. The assumptions on $B$, along with Theorem 5.3, allow us to choose $\mu^{\prime} \in \mathcal{B}$ such that $b:=\operatorname{sh}_{\mu^{\prime}}(B)>0$. Consider the algebra $\mathfrak{A}_{\text {bdd }}$ of all bounded subsets of $\mathbb{R}$. Define the set function $\mu$ on $\mathfrak{A}_{\text {bdd }}$ by means of the equality $\mu(S)=(1 / b) \mu^{\prime}(S \cap B)$ for each $S \in \mathfrak{A}_{\mathrm{bdd}}$. Extend $\mu$ to a measure $\nu$ defined 
on all subsets of $\mathbb{R}$ by means of the equality

$$
\nu(X)=\sum_{i \in \mathbb{Z}} \mu(X \cap[i, i+1)) .
$$

Clearly, $\nu$ is well defined, since $\mu^{\prime}$ is defined on all subsets of $\mathbb{R}$ and $b>0$. To see that $\nu$ extends Lebesgue measure, first note that for any bounded $\lambda$-measurable set $E$, we have

$$
\nu(E)=\frac{1}{b} \mu^{\prime}(E \cap B)=\frac{1}{b}\left(\operatorname{sh}_{\mu^{\prime}} B\right) \mu(E)=\lambda(E)
$$

so $\nu$ and $\lambda$ agree on the bounded $\lambda$-measurable sets. Using (3) and the $\sigma$-finiteness of $\lambda$, we see that $\nu$ and $\lambda$ agree on the unbounded $\lambda$-measurable sets as well.

As to invariance, if $g$ is any isometry on $\mathbb{R}$ and $S \subset \mathbb{R}$ is bounded, we have $\mu(g(S))=(1 / b) \mu^{\prime}(B \cap g(S))=(1 / b) \mu^{\prime}\left(g^{-1}(B) \cap S\right)=(1 / b) \mu^{\prime}(B \cap S)=$ $\mu(S)$, showing that $\mu$ is isometry-invariant on $\mathfrak{A}_{\text {bdd }}$. (Observe that we used the shade-almost invariance of $B$ and the boundedness of $S$ to assert that $\mu^{\prime}\left(g^{-1}(B) \cap S\right)=\mu^{\prime}(B \cap S)$.) The invariance of $\nu$ follows from its definition in terms of $\mu$, so we have shown that $\nu \in \mathcal{B}$. Finally, as to the $\nu$-shade of $B$, observe that for any bounded $\lambda$-measurable $E$ we have $\nu(E \cap B)=$ $\mu(E \cap B)=(1 / b) \mu^{\prime}(E \cap B)$, so (4) shows that $\operatorname{sh}_{\nu}(B)=1$, completing the proof of Lemma 6.1.

We only need finite sequences in the following lemma, but the proof goes through for all denumerable sequences as well.

Lemma 6.2. Let $\left\{t_{0}, t_{1}, t_{2}, \ldots\right\} \subset[0,1]$ be a sequence (finite or denumerable) such that $\sum_{i} t_{i}=1$ and let $B_{0}, B_{1}, B_{2}, \ldots$ be shade-almost invariant subsets of $\mathbb{R}$ such that the following conditions hold:

(i) the $B_{i}$ are pairwise shade-almost disjoint;

(ii) none of the $B_{i}$ has shade zero.

Then there exists a $\mu \in \mathcal{B}$ for which each of the $B_{i}$ is a $\mu$-shading with $\operatorname{sh}_{\mu}\left(B_{i}\right)=t_{i}, i=0,1,2, \ldots$

Proof. For each $i$, apply Lemma 6.1 and condition (ii) to obtain $\mu_{i} \in \mathcal{B}$ such that $\operatorname{sh}_{\mu_{i}}\left(B_{i}\right)=1$ for each $i$. The shade-almost disjointness of the $B_{i}$ implies that $\operatorname{sh}_{\mu_{i}}\left(B_{j}\right)=0$ for each $i \neq j$. It follows that if we define the function $\mu$ on $2^{\mathbb{R}}$ by means of $\mu(S)=\sum_{i} t_{i} \mu_{i}(S)$ for each $S \subset \mathbb{R}$, then $\mu$ is in $\mathcal{B}$ and satisfies the conclusion of the lemma.

7. Shadings from Hamel bases. Let $H$ be any Hamel basis for $\mathbb{R}$ over $\mathbb{Q}$, injectively well-ordered as $\left\{h_{\alpha}: \alpha<c\right\}$. Consider $H$ to be fixed until otherwise stated. 
Each $x \in \mathbb{R}$ has a unique representation as

$$
x=\sum_{\alpha<c} q_{\alpha}(x) h_{\alpha},
$$

where $q_{\alpha}(x) \in \mathbb{Q}$ and all but finitely many of the $q_{\alpha}(x)$ are zero. We may think of $q_{\alpha}$ as the projection of $x$ onto the $\alpha$ th axis of the infinite-dimensional vector space $\mathbb{R}$ over $\mathbb{Q}$ (with basis $H$ ). (Few would call $q_{\alpha}$ a true projection, as it is not linear in the strict sense. The function $q_{\alpha}: \mathbb{R} \rightarrow \mathbb{R}$ is, however, an additive function.)

The next definition plays a key role in what follows.

Definition 7.1. For $K \subset H$, let $W(K)$ denote the set of all reals whose expansion (5) with respect to $H$ involves no members of $K$, i.e.,

$$
W(K)=\left\{x \in \mathbb{R}: q_{\alpha}(x)=0 \forall h_{\alpha} \in K\right\} .
$$

Another way to put it is that $W(K)$ is the linear span or hull (over the rationals) of the basis elements $H \backslash K$. For $K$ given explicitly as a finite sequence $\left\{k_{0}, k_{1}, \ldots, k_{n}\right\}$ we drop the braces and simply write $W\left(k_{0}, k_{1}, \ldots, k_{n}\right)$ for $W(K)$. We will employ a few other notations for convenience. For a sequence $Q_{0}, Q_{1}, \ldots, Q_{n}$ of subsets of rationals, let

$$
A\left(Q_{0}, Q_{1}, \ldots, Q_{n}\right)=W\left(h_{0}, h_{1}, \ldots, h_{n}\right)+Q_{0} h_{0}+Q_{1} h_{1}+\cdots+Q_{n} h_{n} .
$$

Thus $x \in A\left(Q_{0}, Q_{1}, \ldots, Q_{n}\right)$ if and only if $q_{\alpha}(x) \in Q_{\alpha}$ for each $\alpha \in[0, n]$. Let $A^{+}=A\left(\mathbb{Q}^{+}\right)$, where $\mathbb{Q}^{+}$is the set of (strictly) positive rationals, and similarly define $A^{-}=A\left(\mathbb{Q}^{-}\right)$, where $Q^{-}=-Q^{+}$. (Our sequences $\left\{Q_{\alpha}\right\}$ are finite, though transfinite ones still make sense.)

Lemma 7.2. The set $W(K)$ has shade zero whenever $K$ is nonempty.

The preceding lemma follows from [10, Example 4.8]. Something a bit stronger is proved next, in Lemma 7.3 .

While it is not needed here, it is interesting to note that for each $K \subset H$, the set $W(K)$ is homogeneous in the sense of Emil Borel [1: $X \subset \mathbb{R}$ is homogeneous if whenever $x, y \in X$, then $X+x-y=X$. Erdôs and Marcus show in [5, Lemma 2] that every nontrivial homogeneous set has shade zero, from which Lemma 7.2 follows. (Of course, the term "shade" was not used in [5].)

It is easy to show that $A^{+}$has shade $1 / 2$. (This is mentioned in [10, Example 4.8]; the proof is easy.) Another useful fact is the following

Lemma 7.3. If $Q \subset \mathbb{Q}$ is bounded and $h_{\alpha} \in H$, the set $\left\{x: q_{\alpha}(x) \in Q\right\}$ has shade zero.

Proof. It suffices to prove this for $\alpha=0$. Then $\left\{x: q_{\alpha}(x) \in Q\right\}=A(Q)$. We may assume $Q \subset(r, s) \cap \mathbb{Q}$ for rationals $r<s$. Let $Q_{r}=(-\infty, r) \cap \mathbb{Q}$ and $Q_{s}=(s, \infty) \cap \mathbb{Q}$. Then $A\left(Q_{s}\right)=s h_{0}+A\left(\mathbb{Q}^{+}\right)$and $A\left(Q_{r}\right)=r h_{0}-A\left(\mathbb{Q}^{+}\right)$, 
so the invariance properties of Banach measures imply that $\operatorname{sh}\left(A\left(Q_{r}\right)\right)=$ $\operatorname{sh}\left(A\left(Q_{s}\right)\right)=1 / 2$. Therefore, since the sets $A(Q), A\left(Q_{r}\right)$ and $A\left(Q_{s}\right)$ are disjoint, it follows that for any $\mu \in \mathcal{B}$ and any bounded $E \in \mathfrak{L}$, we have

$$
\begin{aligned}
\mu(E \cap A(Q)) & \leq \mu(E)-\mu\left(E \cap A\left(Q_{r}\right)\right)-\mu\left(E \cap A\left(Q_{s}\right)\right) \\
& =\mu(E)-\frac{1}{2} \mu(E)-\frac{1}{2} \mu(E)=0 .
\end{aligned}
$$

We have used translation and reflection invariance of Banach measures in the preceding proof, but we note that the result holds using only translation invariance: Since $Q \subset \mathbb{Q}$ is bounded, there exist countably many disjoint translates $Q+q_{k}$ of $Q$ with each translator $q_{k}$ rational. The sets $q_{k} h_{0}+A(Q)$ are disjoint, congruent and Archimedean, hence each has shade zero.

Intuitively, one might think of $A^{+}$(and similarly, $A\left(Q_{r}\right), A\left(Q_{s}\right)$ ) as being analogous to a "half-plane", or perhaps more aptly, the space of reals lying on one side of a "hyperplane" in an infinite-dimensional Hamelbild of the reals. In this way, it makes some sense that the reals are "evenly split", forming a $1 / 2$-shading. Likewise, when $Q$ is bounded, the reals in $A(Q)$ might be thought of as lying in a "strip" bounded by two such parallel hyperplanes, and so have an arbitrarily small measure relative to the entire space. (We shall subsequently see that further extension of such geometric analogies can be unwise.)

Continuing along these lines, let us employ notations of the form $A^{*+}$, $A^{+--}, A^{++*-}$, etc., so that,,$+- *$ correspond to the sets of rationals $\mathbb{Q}^{+}$, $\mathbb{Q}^{-}, \mathbb{Q}$, respectively. So, for instance, $A^{+*-}$ represents $A\left(\mathbb{Q}^{+}, \mathbb{Q}, \mathbb{Q}^{-}\right)$, the set of real $x$ whose coefficients $q_{0}(x)$ and $q_{2}(x)$ in (5) are, respectively, positive and negative, and the coefficient $q_{1}(x)$ is arbitrary. Clearly, trailing asterisks can be omitted: $A^{+*-}=A^{+*-*}=A^{+*-* *}$, etc.

Now consider the set $A^{++}$. One might suspect that this set must have shade $1 / 4$, as it is the intersection of $A^{+*}\left(=A^{+}\right)$and $A^{*+}$, each of which has shade $1 / 2$. In terms of hyperplanes, one might be tempted to imagine this set as a "quadrant" of a Hamel-based vector space. However, to dispel such a notion, let us be reminded not to imagine Hamel basis vectors as being in any way "mutually orthogonal" $\left({ }^{1}\right)$. The same can be said of the eight sets $A^{ \pm \pm \pm}$_one might think that they should each have shade $1 / 8$. Each of these eight sets is Archimedean with a dense set of translators given by $W\left(h_{0}, h_{1}, h_{2}\right)$, so each has some constant $\mu$-shade for each fixed $\mu \in \mathcal{B}$. For such $\mu$ the $\mu$-shade of any of $A^{ \pm \pm}, A^{ \pm \pm \pm}$, etc., can be no greater than $1 / 2$, however, other than that restriction, we will see in Section 8 that any $\mu$-shades for these sets are possible.

$\left.{ }^{1}\right)$ This point was made to the author by Alexander Kharazishvili during the 24th Summer Symposium in Real Analysis at the University of North Texas. 
Meanwhile, it is immediately clear that it is impossible that all eight of the sets $A^{ \pm \pm \pm}$have shade zero, or even $\mu$-shade zero for some particular $\mu$. To assume otherwise, it follows by finite additivity that the union of all eight sets has $\mu$-shade zero as well. But this union is the complement in $\mathbb{R}$ of $W\left(h_{0}, h_{1}, h_{2}\right)$, which does have shade zero, and we would be forced to conclude that the reals have shade zero, contrary to the obvious fact that $\operatorname{sh}(\mathbb{R})=1$. What is less obvious (but intuitively reasonable) is that none of the eight sets $A^{ \pm \pm \pm}$has shade zero. We will prove this in Section 8 .

Definition 7.4. Fix a set of ordinals $M \subset[0, c)$ and to each $\alpha \in M$ associate a sign $\sigma(\alpha) \in\{-1,1\}$. Denote by $A_{M, \sigma}$ the set of all $x \in \mathbb{R}$ for which, in the Hamel expansion (5) of $x, \sigma(\alpha) q_{\alpha}(x)>0$ for all $\alpha \in M$. Notice that $A_{M,-\sigma}=-A_{M, \sigma}$; let $A_{M, \pm \sigma}=A_{M, \sigma} \cup A_{M,-\sigma}$.

This is simply a generalization of sets we have introduced before, more precisely defined (even if less intuitively notated). As an example, let $M=$ $\{1,3\}, \sigma(1)=1$, and $\sigma(3)=-1$. Then $A_{M, \sigma}=A^{*+*-}=A\left(\mathbb{Q}, \mathbb{Q}^{+}, \mathbb{Q}, \mathbb{Q}^{-}\right)$, which is the set of reals whose Hamel basis expansions have positive coefficients of $h_{1}$ and negative coefficients of $h_{3}$, and $A_{M, \pm \sigma}=A^{*+*-} \cup A^{*-*+}$.

Notice that, in general, $-A_{M, \pm \sigma}=A_{M, \pm \sigma}$ and that $A_{M, \sigma}$ and $A_{M,-\sigma}$ are Archimedean with a dense set of translators being $W\left(\left\{h_{\alpha}: \alpha \in M\right\}\right)$. But it is translations by the rest of the reals that give such sets interesting properties, as we see in the next result.

Lemma 7.5. The sets $A_{M, \pm \sigma}$ in Definition 7.4 are shade-almost invariant.

Proof. Fix $M$ and $\sigma$. The result is trivial if $M=\emptyset$, so we may assume otherwise. Let $A$ and $W$ denote $A_{M, \sigma}$ and $W\left(\left\{h_{\alpha}: \alpha \in M\right\}\right)$, respectively. We first show that that $t+A \doteqdot A$ for any $t \in \mathbb{R}$. We already noted that if $t \in W$, then we have $t+A=A$, so suppose that $t \notin W$. It suffices to show that $(t+A) \triangle A$ is contained in a finite union of the "strips" alluded to previously. Specifically, since $t \notin W$ we can write $t=\sum_{\alpha \in M^{\prime}} r_{\alpha} h_{\alpha}+w$ for some $w \in W$ and rational $r_{\alpha} \neq 0$, where $M^{\prime}$ is a finite subset of $M$ (since the Hamel expansion of $t$ involves only finitely many $\alpha$ ). Then $(t+A) \triangle A \subset \bigcup_{\alpha \in M^{\prime}}\{x$ : $\left.q_{\alpha}(x) \in Q_{\alpha}\right\}$, where $Q_{\alpha}=\mathbb{Q} \cap\left(0, r_{\alpha}\right]$ if $r_{\alpha}>0$ and $Q_{\alpha}=\mathbb{Q} \cap\left[r_{\alpha}, 0\right)$ if $r_{\alpha}<0$. It follows from Lemma 7.3 that $(t+A) \triangle A \doteqdot \emptyset$.

That takes care of translations. Looking at reflections, it is now clear that for any $t \in \mathbb{R}, t-A=-((-t)+A) \doteqdot-A$. This means that for any isometry $g$ on $\mathbb{R}$ we have $g(A \uplus(-A)) \doteqdot A \uplus(-A)$.

8. Some custom-built Banach measures. The results in this section can probably be generalized a great deal, but we settle for the situation at 
hand pertaining to our particular Archimedean sets. Refer to Definitions 7.1 and 7.4 for what follows.

In this section $G$ denotes the group of all isometries on $\mathbb{R}, M$ is a fixed subset of $[0, c)$ with $2 \leq|M| \leq \aleph_{0}$, and $A=A_{M, \pm \sigma}$.

Obviously $A$ and $A^{\mathrm{c}}$ cannot both have shade zero, but the main hurdle in what follows is to show that neither can have shade zero. To prove this we will find a Banach measure $\mu$ with respect to which each of $A$ and $A^{\mathrm{c}}$ has positive shade, i.e., $\operatorname{sh}_{\mu}(A)>0$ and $\operatorname{sh}_{\mu}\left(A^{\mathrm{c}}\right)>0$.

Definition 8.1. For $\alpha \in M$, consider sets of the form $W\left(h_{\alpha}\right)+Q_{\alpha} h_{\alpha}$, where each $Q_{\alpha}$ is a bounded subset of $\mathbb{Q}$. Define $\mathcal{T}_{M}$ to be the ideal generated by all such $W\left(h_{\alpha}\right)+Q_{\alpha} h_{\alpha}, \alpha \in M$.

Each $W\left(h_{\alpha}\right)+Q_{\alpha} h_{\alpha}$ in Definition 8.1 is a "Hamel cylinder" of sorts, contained in one of our "strips" mentioned earlier. It is clear by Lemma 7.3 that each $W\left(h_{\alpha}\right)+Q_{\alpha} h_{\alpha}$ has shade zero, so $\mathcal{T}_{M} \subset \mathcal{Z}$.

TheOREM 8.2. Let $\theta \in[0,1]$. Then there exists a Banach measure $\mu_{\theta}$ for which $\operatorname{sh}_{\mu}(A)=\theta$.

Proof. Note that $-A=A$, since $-A_{M, \sigma}=A_{M,-\sigma}$. The following clearly hold:

(i) $g(A) \triangle A=g\left(A^{\mathrm{c}}\right) \triangle A^{\mathrm{c}} \in \mathcal{T}_{M}$ for any $g \in G$;

(ii) $A \notin \mathcal{T}_{M}$ and $A^{\mathrm{c}} \notin \mathcal{T}_{M}$.

The proof of Lemma 7.5 implies property (ii), which says that $A$ and (therefore) $A^{\mathrm{c}}$ are $\mathcal{T}_{M}$-almost invariant. Property (ii) holds since any member of $\mathcal{T}_{M}$ differs from $A$ and $A^{\mathrm{c}}$ by sets of reals $x$ having arbitrarily large values of $\left|q_{\alpha}(x)\right|$ for each $\alpha \in M$. It is straightforward to verify that the family $G(A)$ of all translates and reflections of $A$ generates a $G$-invariant algebra $\mathfrak{A}(A)$ whose members are sets having one of the forms

$$
N_{1}, \quad\left(A \backslash N_{1}\right) \cup N_{2}, \quad\left(A^{\mathrm{c}} \backslash N_{1}\right) \cup N_{2}, \quad \mathbb{R} \backslash N_{1} \quad\left(N_{1}, N_{2} \in \mathcal{T}_{M}\right) .
$$

This allows us to define a finitely additive $G$-invariant probability measure $\nu_{\theta}$ on $\mathfrak{A}(A)$ by means of the assignments

$\nu_{\theta}\left(N_{1}\right)=0, \nu_{\theta}\left(\left(A \backslash N_{1}\right) \cup N_{2}\right)=\theta, \quad \nu_{\theta}\left(\left(A^{\mathrm{c}} \backslash N_{1}\right) \cup N_{2}\right)=1-\theta, \nu_{\theta}\left(\mathbb{R} \backslash N_{1}\right)=1$, where $\theta$ is any fixed number in $[0,1]$. That $\nu_{\theta}$ is well defined can be verified directly using the aforementioned properties of $\mathcal{T}_{M}$.

For the convenience of directly applying a result of Marczewski concerning products of probability measures, we define the probability measure space $\left(\mathfrak{L}_{0}, \lambda_{0}\right)$ as follows. Let $\mathfrak{L}_{0}=\left\{\mathbb{Z}+E_{0}: E_{0} \in \mathfrak{L}\right.$ and $\left.E_{0} \subset[0,1)\right\}$, and define $\lambda_{0}\left(\mathbb{Z}+E_{0}\right)=\lambda\left(E_{0}\right)$. (The space $\left(\mathfrak{L}_{0}, \lambda_{0}\right)$ is, in essence, the Lebesgue measure space on $[0,1)$.) We now show that the probability measure spaces $\left(\mathfrak{A}(A), \nu_{\theta}\right)$ 
and $\left(\mathfrak{L}_{0}, \lambda_{0}\right)$ are almost independent in the sense of Marczewski [11, meaning that if $X \in \mathfrak{A}(A)$ and $E_{0} \in \mathfrak{L}_{0}$ with $\nu_{\theta}(X) \neq 0$ and $\lambda_{0}\left(E_{0}\right) \neq 0$, then $X \cap E_{0} \neq \emptyset$.

To see this, let $\beta \in[0, c) \backslash M$ and let $H_{M}=\left\{h_{\beta}\right\} \cup\left\{h_{\alpha}: \alpha \in M\right\}$. (The added element $h_{\beta}$ will be used only to simplify a portion of the proof ahead.) First notice that if $X \in \mathfrak{A}(A)$ with $\nu_{\theta}(X)>0$, then $X=P_{M}+W_{M}$, where $W_{M}$ is the span (over the rationals) of $H \backslash H_{M}, P_{M}$ is countable (formed of certain linear combinations, over $\mathbb{Q}$, of the set of basis elements $H_{M}$ ), and $P_{M}$ is dense in $\mathbb{R}$ (obvious, thanks to the addition of $h_{\beta}$ ). Notice also that $\mathbb{R}=Q_{M}+W_{M}$, where $Q_{M}$ is the set (still countable) of all linear combinations, over $\mathbb{Q}$, of the basis elements $H_{M}$. This shows that the reals are covered by countably many translates of $W_{M}$, so we know that $W_{M}$ cannot have measure zero. We will use the following well-known fact (it follows from the Lebesgue density theorem).

Lemma 8.3. Let $E \subset \mathbb{R}$ be a set of positive Lebesgue measure. Then $\lambda(\mathbb{R} \backslash(E+T))=0$ whenever $T$ is a dense set.

Now suppose that $E \cap X=\emptyset$, where $X$ is as postulated just earlier and $E=\mathbb{Z}+E_{0}$ is a set of positive measure in $\mathfrak{L}_{0}$ (equivalently, $E_{0}$ is a set of positive Lebesgue measure in $[0,1))$. Then $E \cap\left(x+W_{M}\right)=\emptyset$ for each $x \in P_{M}$, hence $(E-x) \cap W_{M}=\emptyset$ for each such $x$. But this means that $\left(E-P_{M}\right) \cap W_{M}$ is empty. Since $E$ has positive Lebesgue measure, it follows from Lemma 8.3 that $E-P_{M}=\mathbb{R} \backslash N$ for some set $N$ of Lebesgue measure zero. But then, since $\left(E-P_{M}\right) \cap W_{M}=\emptyset$, it follows that $W_{M} \subset N$, implying $W_{M}$ has Lebesgue measure zero, which we already know not to be the case. This contradiction finally establishes that $E \cap X \neq \emptyset$, and in turn that $\left(\mathfrak{A}(A), \nu_{\theta}\right)$ and $\left(\mathfrak{L}_{0}, \lambda_{0}\right)$ are almost independent measure spaces.

This enables us to define a measure $\mu_{0}$ on the algebra $\mathfrak{A}_{0}(A)$ generated by $\mathfrak{A}(A) \cup \mathfrak{L}_{0}$ that is the multiplicative extension of $\nu_{\theta}$ and $\lambda_{0}$. (See Theorem I in [11].) In particular, we have

$$
\mu_{0}\left(A \cap\left(\mathbb{Z}+E_{0}\right)\right)=\nu_{\theta}(A) \lambda_{0}\left(\mathbb{Z}+E_{0}\right)=\theta \lambda\left(E_{0}\right)
$$

when $\mathbb{Z}+E_{0} \in \mathfrak{L}_{0}$.

Note that $\left(\mathfrak{A}_{0}(A), \mu_{0}\right)$ is a $G$-invariant measure space. The algebra $\mathfrak{A}^{\prime}(A)$ generated by $\mathfrak{A}(A) \cup \mathfrak{L}$ consists of sets of the form $\biguplus_{k \in \mathbb{Z}} Y_{k} \cap[k, k+1)$, where $Y_{k} \in \mathfrak{A}_{0}(A)$ for each $k$. We can then define the $G$-invariant measure $\mu^{\prime}$ on $\mathfrak{A}^{\prime}(A)$ by means of the equality

$$
\mu^{\prime}\left(\biguplus_{k \in \mathbb{Z}}\left(Y_{k} \cap[k, k+1)\right)\right)=\sum_{k \in \mathbb{Z}} \mu_{0}\left(Y_{k}\right) .
$$

Clearly, the measure $\mu^{\prime}$ is well defined, extends the Lebesgue measure, is $G$-invariant, and has the property that $\mu^{\prime}(A \cap E)=\theta \lambda(E)$ for any $E \in \mathfrak{L}$. Since $\mu^{\prime}$ is $G$-invariant, it can be extended (via the Invariant Extension 
Theorem [19, Thm. 10.8]) to a Banach measure $\mu$. This, at last, proves that the set $A$ is a $\mu$-shading with $\operatorname{sh}_{\mu}(A)=\theta$.

COROLlary 8.4. The set $A_{M, \sigma}$ cannot have shade zero for any choice of $\sigma$. In particular, none of the eight sets $A^{ \pm \pm \pm}$has shade zero.

Proof. By choosing $\theta \in(0,1)$, we see that neither $A$ nor $A^{\mathrm{c}}$ can be a set of shade zero. Since $\operatorname{sh}_{\mu}(A)=2 \operatorname{sh}_{\mu}\left(A_{M, \sigma}\right)$, the conclusion follows.

We note that if $|M|=1$, then $\operatorname{sh}\left(A_{M, \sigma}\right)=1 / 2$. Also, despite warnings that, for example, $\operatorname{sh}_{\mu}\left(A^{++} \cup A^{--}\right)$need not equal $1 / 2$, it is clear that this does indeed happen for certain $\mu$. Likewise, toward the other extreme, if $|M|=\aleph_{0}$, then despite the seemingly thinned-out nature of any resulting $A_{M, \sigma}$ (being akin to the set $\mathbb{R}$ cut by half denumerably many times), such sets still do not have shade zero, though they can have $\mu$-shade zero (or one). In fact, if one wishes to consider even larger $M$, even with $|M|=c$ (or to simply shorten the proof of Theorem 8.2, avoiding the paragraphs surrounding Lemma 8.3), one can consider suitably tailored Hamel bases that are Bernstein sets for obtaining the independence needed for applying Marczewski's theorem. (Such $H$ do exist and are called Burstin sets [12].)

9. A Hamel basis with a weak closure property. To prove our main result we find a way to control how our $A_{i}$ are stretched. We do so by controlling certain multiplicative properties of the underlying Hamel basis $H$. (See [4, Thm. 2.1/Lemma 2.2] for another application of products in Hamel bases to invariant extensions of Lebesgue measure.) Up to now, no special properties of $H$ have been required, but in this section we impose restrictions on $H$ that we leave in place until the end of the paper.

We will use a result ([16, Theorem 2.1.1]) that is possibly well known and whose proof is straightforward enough to omit. We note first that if $\mathbb{E}$ is an algebraic subfield of a field $\mathbb{F}$, then $\mathbb{F}$ always has a Hamel basis over $\mathbb{E}$. (The proof of this fact is also routine.)

Lemma 9.1. For $n \geq 2$ let $\mathbb{F}_{0} \subsetneq \mathbb{F}_{1} \subsetneq \cdots \subsetneq \mathbb{F}_{n}$ be a properly inclusive sequence of algebraic fields (i.e., a tower), and let $H_{i}$ be a Hamel basis for the field $\mathbb{F}_{i}$ over the proper subfield $\mathbb{F}_{i-1}$ for $i=1, \ldots, n$. Then the set of products

$$
H=\prod_{i=1}^{n} H_{i}=\left\{h_{1} \cdots h_{n}: h_{i} \in H_{i}\right\}
$$

is a Hamel basis for $\mathbb{F}_{n}$ over $\mathbb{F}_{0}$.

We apply the lemma with $n=2$ as follows. From now on, let $\mathbb{F}_{2}=\mathbb{R}$, $\mathbb{F}_{1}=\mathbb{Q}[\sqrt[3]{2}]$, and $\mathbb{F}_{0}=\mathbb{Q}$. ( $\mathbb{F}_{1}$ is the smallest field containing $\mathbb{Q}$ and $\sqrt[3]{2}$.) 
Then

$$
H_{1}=\{1, \sqrt[3]{2}, \sqrt[3]{4}\}
$$

is a basis for $\mathbb{F}_{1}$ over $\mathbb{Q}$; for each member $e$ of $\mathbb{F}_{1}$ there are unique rationals $q_{0}, q_{1}, q_{2}$ for which $e=q_{0} \cdot 1+q_{1} \cdot \sqrt[3]{2}+q_{2} \cdot \sqrt[3]{4}$. Let $H_{2}$ be any basis for $\mathbb{R}$ over $\mathbb{F}_{1}$ for which $1 \in H_{2}$ (this is always possible). We will henceforth let

$$
H=H_{1} H_{2}
$$

denote the Hamel basis for $\mathbb{F}_{2}=\mathbb{R}$ over $\mathbb{F}_{0}=\mathbb{Q}$ whose existence is asserted in Lemma 9.1. Notice that we have $H_{1} \subset H$. In our injective well-ordering $\left\{h_{\alpha}\right\}_{\alpha<c}$ of $H$, we may and do assume that

$$
h_{0}=1, \quad h_{1}=\sqrt[3]{2}, \quad h_{2}=\sqrt[3]{4},
$$

which we desire for later convenience of notation.

The basis $H_{1}$ has the following convenient property, a weak sort of multiplicative closure:

$$
k, k^{\prime} \in H_{1} \Rightarrow k k^{\prime}=q k^{\prime \prime} \text { for some } q \in \mathbb{Q} \text { and } k^{\prime \prime} \in H_{1} .
$$

We are particularly interested in the products $h_{1} h$ for $h \in H$. If $h \in H_{1}=$ $\left\{h_{0}, h_{1}, h_{2}\right\}$, this amounts to the cyclicity:

$$
h_{1} h_{0}=h_{1}, \quad h_{1}^{2}=h_{2}, \quad h_{1} h_{2}=2 h_{0} .
$$

Otherwise, for $h \in H \backslash H_{1}$, we see that $h_{1} h$ is itself a member of $H \backslash H_{1}$, so that the expansion of $h_{1} h$ involves no members of $H_{1}$. That is,

$$
q_{\alpha}\left(h_{1} h\right)=0 \quad \text { for } \alpha \in\{0,1,2\} \text { and } h \in H \backslash H_{1} .
$$

(Recall that $q_{\alpha}$ is defined by (5).) What must be emphasized is that the expansion of $\sqrt[3]{2} h$ involves no member of $H_{1}$ for $h \notin H_{1}$, while the expansion of $\sqrt[3]{2} h$ involves only members of $H_{1}$ when $h \in H_{1}$. Furthermore, $q_{\alpha}(\sqrt[3]{2} h) \geq 0$ for $\alpha \in\{0,1,2\}$ and $h \in H_{1}$.

For the rest of this paper, let

$$
A_{0}=A^{*++} \cup A^{*--}, \quad A_{1}=A^{+*+} \cup A^{-*-}, \quad A_{2}=A^{++*} \cup A^{--*} .
$$

(See Section 7 for the relevant definitions.)

By regarding the properties just mentioned, with this basis $H$ our sets $A_{0}, A_{1}, A_{2}$ enjoy the following cyclical properties under stretching by the factor of $\sqrt[3]{2}$ :

$$
\sqrt[3]{2} A_{0}=A_{1}, \quad \sqrt[3]{2} A_{1}=A_{2}, \quad \sqrt[3]{2} A_{2}=A_{0} .
$$

We establish some other useful properties of $A_{0}, A_{1}, A_{2}$ in Section 10 .

10. A Banach measure that is not scale-invariant. The main motivation for this paper is the following theorem.

TheOREm 10.1. There exists a Banach measure $\mu$, an Archimedean set $A$ (thereby a $\mu$-shading), and $h \in \mathbb{R}$ such that $\operatorname{sh}_{\mu}(h A) \neq \operatorname{sh}_{\mu}(A)$. 
The proof of Theorem 10.1 will be easy once we establish some useful properties of the sets $A_{0}, A_{1}, A_{2}$ defined by (7) in Section 9. By Lemma 7.5. each of these three sets is shade-almost invariant. Each is of the form $A_{M, \pm \sigma}$ with $|M|=2$, so by Theorem 8.2 none has shade zero.

We can now construct a measure having convenient properties on $A_{0}, A_{1}, A_{2}$.

LEMmA 10.2. Let $t_{0}, t_{1}, t_{2}, t_{3}$ be any real numbers chosen from $[0,1]$ such that $t_{0}+t_{1}+t_{2}+t_{3}=1$. Then there exists a Banach measure $\mu$ for which $\operatorname{sh}_{\mu}\left(A_{i}\right)=t_{i}+t_{3}$ for $i=0,1,2$.

Proof. Let $B_{0}=A^{-++} \cup A^{+--}, B_{1}=A^{+-+} \cup A^{-+-}, B_{2}=A^{++-} \cup A^{--+}$, $B_{3}=A^{+++} \cup A^{---}$. Then $B_{0}, B_{1}, B_{2}, B_{3}$ have the following properties.

(i) The sets $B_{0}, B_{1}, B_{2}, B_{3}$ are pairwise shade-almost disjoint. (They are pairwise disjoint.)

(ii) None of $B_{0}, B_{1}, B_{2}, B_{3}$ has shade zero. (This follows from Corollary 8.4 .)

Therefore, by Lemma 6.2 there is a Banach measure $\mu$ for which $\operatorname{sh}_{\mu}\left(B_{i}\right)=t_{i}$ for $i=0,1,2,3$. Finally, for $i=0,1,2$, the set $A_{i}$ is the shade-almost disjoint union of $B_{i}$ and $B_{3}$, hence $\operatorname{sh}_{\mu}\left(A_{i}\right)=\operatorname{sh}_{\mu}\left(B_{i} \cup B_{3}\right)=t_{i}+t_{3}$.

We do not need it here, but the following answers a question posed in [10, Problem 3].

COROLlary 10.3. There exist Archimedean sets which are not shadings.

Proof. The sets $A_{0}, A_{1}, A_{2}$ serve as examples, since Lemma 10.2 shows that their $\mu$-shades depend on the particular $\mu$.

The set $C+\mathbb{Q}$ mentioned earlier also serves as such an example, as would any shade-almost invariant Archimedean set that does not have shade zero or one.

Proof of Theorem 10.1. With $H$ the Hamel basis constructed in Section 9 , let $A=A_{2}$ and $h=h_{1}=\sqrt[3]{2}$. Letting $\mu$ be the measure obtained in Lemma 10.2 and noting that $h A_{2}=A_{0}$, we find that $\operatorname{sh}_{\mu}\left(A_{2}\right)=t_{2}+t_{3}$ but $\operatorname{sh}_{\mu}\left(\hat{h A_{2}}\right)=\operatorname{sh}_{\mu}\left(A_{0}\right)=t_{0}+t_{3}$, which need not equal $t_{2}+t_{3}$.

It is instructive (or at least, amusing) to consider some extreme examples of Theorem 10.1. For instance, we can let $t_{0}=1$ and $t_{1}=t_{2}=t_{3}=0$, so that for the corresponding $\mu$ constructed via Theorem 10.1, we have $\operatorname{sh}_{\mu}\left(A_{2}\right)=0$ while $\operatorname{sh}_{\mu}\left(h A_{2}\right)=1$. Also, it is not difficult to see that for any nonzero rational $q$, the $\mu$-shade of the $A_{i}$ is equal to that of $q A_{i}$ (for any $\mu$ ). Therefore, $q h$ can be any size, so that $q h A_{2}$ can be either a dilation or a contraction of $A_{2}$. And $q h$ can be arbitrarily close to 1 , showing that a stretch (or contraction) 
by the smallest amount can still give an extreme change in the $\mu$-shade density. Also, using the same example, we can consider the set $A=A_{2} \cap(0, \infty)$, which has (constant) $\mu$-shade zero, whereas $h A$ has $\mu$-shade equal to 1 on $(0, \infty)$ but 0 on $(-\infty, 0)$. Scandalous!

Such possibilities were alluded to in the abstract: a similarity transformation of a set of constant $\mu$-shade might not have constant $\mu$-shade. How far can this behavior be stretched? Employing other extension fields of $\mathbb{Q}$, such as $\mathbb{Q}[\sqrt[n]{2}]$ for large $n$, more fun (or mockery) can certainly be had, by allowing greater varieties of $\mu$-shades to result from a single transformation (with different $\mu$ ). In this way, given an arbitrary step function $f$ with values in $[0,1]$ and only finitely many jumps, one can construct a set $B \subset \mathbb{R}$ and Banach measure $\mu$ for which $\operatorname{sh}_{\mu}(B)=1 / 2$ (say) but $\operatorname{sh}_{\mu}(x B)=f$ for some $x>0$. By considering different fields and/or building "taller towers" in the construction of the Hamel bases via Lemma 9.1, one can get infinitely many basis elements into the act (since Lemma 6.2 holds for countably many $B_{i}$ ). It should then be possible to pass from step functions to continuous functions, using techniques similar to those used for creating continuously varying shadings in [10, Thm. 5.7] or [14, Thm. 4.1].

How far can this go? Is it possible that given two different continuous $f_{1}, f_{2}: \mathbb{R} \rightarrow[0,1]$ there exists a set $B \subset \mathbb{R}$, a Banach measure $\mu$, and $x>0$ for which $\operatorname{sh}_{\mu}(B)=f_{1}$ and $\operatorname{sh}_{\mu}(x B)=f_{2}$ ? For such $f_{1}$ and $f_{2}$, do there exist Banach measures $\mu_{1}$ and $\mu_{2}$ for which $\operatorname{sh}_{\mu_{1}}(B)=f_{1}$ and $\operatorname{sh}_{\mu_{2}}(B)=f_{2}$ ? If these sorts of examples are possible, can one go further and devise sets that are more universal, such as a single set $B$ for which there is a Banach measure $\mu$ such that for each continuous $f$ there is an $x$ for which $\operatorname{sh}_{\mu}(x B)=f$ ?

In all of this fun, we must bear in mind that these sets have $\mu$-shades for particular $\mu \in \mathcal{B}$, as opposed to all Banach measures. It merely shows that these Banach measures are not able to agree on the density of these sets. In that respect, these measures are "not very good" at measuring such sets, one (e.g., [3]) might say.

Aside from such diverting examples, we more importantly have shown the following main result.

COROLlary 10.4. There exists a Banach measure on $\mathbb{R}$ that is not scaleinvariant.

Proof. Our measure $\mu$, constant $h$, and set $A$ constructed in Theorem 10.1 witness the statement of the corollary. To see this more explicitly, consider the set $S=A \cap I$, where $I$ is any bounded interval. The corollary follows if $\mu(h S) \neq h \mu(S)$. Indeed, on the one hand we have $\mu(h S)=\mu((h A) \cap(h I))$ $=\operatorname{sh}_{\mu}(h A) \lambda(h I)=\left(\operatorname{sh}_{\mu}(h A)\right) h \lambda(I)$. But on the other hand we have $h \mu(S)=$ $h \mu(A \cap I)=h \operatorname{sh}_{\mu}(A) \lambda(I)$. The equality of these quantities would imply that $\operatorname{sh}_{\mu}(A)=h \operatorname{sh}_{\mu}(A)$, and would then contradict Theorem 10.1. 
11. Conclusion. It now must be pointed out that if $\mu$ is an "improved" Banach measure on $\mathbb{R}$, i.e., one that is scale-invariant, then the $\mu$-shade of a $\mu$-shading is unaffected by a similarity transformation. More precisely, we have the following theorem, the proof of which we leave as an easy exercise for the reader.

THEOREM 11.1. If $\mu$ is an improved Banach measure on $\mathbb{R}$ and $A$ is a $\mu$-shading of $\mathbb{R}$, then $g(A)$ is also a $\mu$-shading and $\operatorname{sh}_{\mu}(g(A))=\operatorname{sh}_{\mu}(A)$ for every similarity transformation $g$ on $\mathbb{R}$.

Speaking of "improved measures" (cf. [2]), it might seem reasonable to incorporate scale-invariance into the definition of a shading, especially in view of Theorem 11.1. After all, reflection-invariance is included even though a similar definition can be given using only translation-invariance. But using translation-invariance alone yields results that are in some fundamental ways unsatisfying. For example, in spite of the fact that $A^{+} \uplus\left(-A^{+}\right) \doteqdot \mathbb{R}$, one cannot claim, without making use of reflection-invariance, that $A^{+}$should be viewed as "half" of the line in any measure-theoretic sense considered here, because translation-invariant total extensions of $\lambda$ can be constructed with respect to which $A^{+}$has any shade (in a similar way to Lemma 6.2 . using the fact that $x+A^{+} \doteqdot A^{+}$for all $x \in \mathbb{R}$ ). Fortunately, we have reflection-invariance available as another inuitively appealing condition. We want our extensions of $\lambda$ to have as many as possible of the properties we consider desirable in $\lambda$, so we include the reflection-invariance in our extensions because - well, because we can. So, if the result of Theorem 10.1 seems unreasonable (especially in view of the remarks following it), we have Theorem 11.1 to settle the matter, and perhaps we should indeed insist upon this improvement in the definition of shadings (because we can). On the other hand, in neither [2] nor [3] is this improvement mentioned, so perhaps tastes will differ in this regard!

Acknowledgements. The author thanks Keith Neu, the author's colleague and former student, for inspiring this article and for his pursuit of and contributions to the theory of shadings, most notably (so far) as in [14 and [15. Thanks also go to Prof. K. Ciesielski for his suggestions and encouragement, and to the author's colleague Tibor Szarvas at LSUS for his reading and discussion of an earlier draft. Finally, the author is grateful for the guidance and patience of an anonymous referee, whose contributions significantly improved the paper.

\section{References}

[1] É. Borel, Les ensembles homogènes, C. R. Acad. Sci. Paris 222 (1946), 617-618.

[2] A. M. Bruckner and J. Ceder, On improving Lebesgue measure, Nordisk Mat. Tidskr. 23 (1975), 59-68. 
[3] K. Ciesielski, How good is Lebesgue measure?, Math. Intelligencer 11 (1989), no. 2, $54-58$.

[4] K. Ciesielski and A. Pelc, Extensions of invariant measures on Euclidean spaces, Fund. Math. 125 (1985), 1-10.

[5] P. Erdôs et S. Marcus, Sur la décomposition de l'espace euclidien en ensembles homogènes, Acta Math. Hungar. 8 (1957), 443-452.

[6] S. Kakutani and J. Oxtoby, Construction of a non-separable invariant extension of the Lebesgue measure space, Ann. of Math. 52 (1950), 580-590.

[7] A. B. Kharazishvili, Invariant Extensions of Lebesgue Measure, Tbilis. Gos. Univ., Tbilisi, 1983 (in Russian).

[8] - Applications of Point Set Theory in Real Analysis, Kluwer, Dordrecht, 1998.

[9] V. Klee, Invariant extension of linear functionals, Pacific J. Math. 4 (1954), 37-46.

[10] R. D. Mabry, Sets which are well-distributed and invariant relative to all isometryinvariant total extensions of Lebesgue measure, Real Anal. Exchange 16 (1990-91), $425-459$.

[11] E. Marczewski, Measures in almost independent fields, Fund. Math. 38 (1951), $217-229$.

[12] A. W. Miller and S. G. Popvassilev, Vitali sets and Hamel bases that are Marczewski measurable, ibid. 166 (2000), 269-279.

[13] J. Mycielski, Finitely additive invariant measures. I, Colloq. Math. 42 (1979), 309-318.

[14] K. Neu, A few results on Archimedean sets, Real Anal. Exchange 34 (2008/2009), 157-170.

[15] —, Almost isometry-invariant sets and shadings, ibid., to appear.

[16] S. Roman, Field Theory, 2nd ed., Springer, New York, 2006.

[17] A. Simoson, An "Archimedean" paradox, Amer. Math. Monthly 89 (1982), 114-116, 125 .

[18] S. Wagon, Invariance properties of finitely additive measures in $\mathbb{R}^{n}$, Illinois J. Math. 25 (1981), 74-86.

[19] —, The Banach-Tarski Paradox, Cambridge Univ. Press, New York, 1985.

Richard D. Mabry

Department of Mathematics

Louisiana State University in Shreveport

Shreveport, LA 71115-2399, U.S.A.

E-mail: Richard.Mabry@LSUS.edu

Received 21 December 2008;

in revised form 3 April 2010 